\title{
15 ADHS und Tiergestützte Therapie
}

Christian Göhre und Dagmar Horn

Die moderne Tiergestützte Therapie wurde 1969 durch Lewinson begründet „The dog as a co-therapist“.

Diese Therapieform ist seit dem in den USA, in Großbritannien, in Österreich sowie in der Schweiz anerkannt und weit verbreitet. Es ist wissenschaftlich erwiesen, dass - ähnlich wie bei den Bindungsverhältnissen zwischen Säuglingen, Kleinkindern und Erwachsenen - eine intensive Bindung zwischen dem Mensch und dem Hund besteht (Frömming 2006).

Dabei wird die Kommunikation zwischen Mensch und Hund als Lernprozess von beiden Seiten verstanden. Oft gelten Tiere auch als Partner von Menschen, die von Behinderungen betroffen sind. Die Hilfe und die Unterstützung des Tieres erfolgt in verschiedenster Art und Weise, wobei sich die Tiergestützte Therapie als ganzheitliches Entwicklungs- und Förderprogramm versteht.

Durch die Tiergestützte Therapie werden das psychische, das soziale und das physische Wirkungsfeld beeinflusst. Ziel ist es, einen positiven Einfluss auf den Genesungsprozess sowie die Verbesserung der Lebensqualität zu erzielen (Greifenhagen \& Buck 2007).

Folgende Einsatzbereiche sind durch die Tiergestützte Therapie zum Beispiel abgesteckt.

- Kliniken

- Behinderten- sowie Altenheime

- Schulen inklusive Sonderschulen

- Einrichtungen der Vorschulerziehung

- Strafvollzug

- Sozialpädagogik 
- Ergotherapie

- Logopädie

Nach Prothmann (2007) erzielt die Tiergestützte Therapie in den drei Hauptarbeitsfeldern folgende Wirkungen:

Physische und physiologische Wirkung

- Senkung des Blutdrucks und der Herzfrequenz, Kreislaufstabilisierung

- Muskelentspannung, Abnahme der Spastik, Besserung des Gleichgewichts

n neuroendokrine Wirkung, Ausschüttung von Endorphinen, Änderung der Schmerzwahrnehmung

- motorische Aktivierung, Training der Muskulatur, Verdauungsaktivierung, Anregung zur besseren Ernährung, bessere Körperpflege, Übergewichtsreduktion, Förderung einer regelmäßigen Tagesstruktur

- Ersatz gestörter Sinnesfunktionen (Blindenhund, Gehörlose, Rollstuhlfahrer)

Psychische Wirkung

- Stabilisierung der Befindlichkeit (Zuneigung, Zärtlichkeit, Trost)

- Förderung von positivem Selbstbild, Selbstwertgefühl und Selbstbewusstsein

- Förderung von Kontrolle über Umwelt und sich selbst

- Förderung von Sicherheit, Selbstsicherheit und Abbau von Angst

- Stressreduktion, Beruhigung und Entspannung

- soziale Integration

- Antidepressive und antisuizidale Wirkung

\section{Soziale Wirkung}

- Aufhebung von Einsamkeit und Isolation

- Nähe, Intimität, Körperkontakt

- Streitschlichtung, Familienzusammenhalt, Rettung der Beziehung

- Positive soziale Attribution

Dass Haustiere allgemein vielfältige entwicklungsfördernde Faktoren für Kinder mit sich bringen, ist jedoch schon vor der Etablierung der Tiergestützten Therapie hinreichend belegt worden (Vernooij \& Schneider 2007, Otterstedt et al. 2004, Olbrich \& von Otterstedt 2003).

Durch den Einsatz von Tieren in Pädagogik und Therapie können sich jedoch entwicklungsfördernde, protektive Faktoren für Kinder und Jugendliche herauskristallisieren und festigen (Röger-Lakenbrink 2006). Nach Prothmann (2007) sind diese entwicklungsfördernden Faktoren wie folgt benannt:

- Verantwortungsgefühl

- Pflichtbewusstsein

- Rücksichtnahme 
- Bedürfnisse anderer respektieren

- Sensibilität sowie Achtung vor Lebewesen

- Freundschaft

- soziale Kompetenz

- Toleranz

- Hilfsbereitschaft

- Gefühle zuzulassen

- Entdeckung der Natur

- Integration in Gemeinschaften

Als multiprofessionell arbeitendes Team einer kinder- und jugendpsychiatrischen Tagesklinik, entschieden wir uns im Jahre 2004 unser therapeutisches Angebot mit der Implementierung der Tiergestützten Therapie zu erweitern. Die Idee, die grundsätzliche Konzeption sowie die Umsetzung, verdanken wir unserem Ärzteehepaar „D. \& S. Horn“, die in unserer Klinik tätig sind. In Gesprächen mit den Mitarbeitern unserer drei Stationen war es uns wichtig, dass wir mindestens auf jeder Station einen Co-Therapeuten aus dem Pflege- und Erziehungsdienst für diese Arbeit begeistern konnten. Hinweise, dass auch das Pflegepersonal von kurzzeitigen Tierkontakten in psychiatrischen Einrichtungen profitieren kann, zeigte die Arbeit von Cassidy et al. (1995).

Danach folgten Schulungen aller Mitarbeiter der Klinik, um das Wissen über Tiergestützte Therapien zu vertiefen und Vorurteile abzubauen.

Unser Therapiebegleithund „Buster“ ist ein drei Jahre alter Labradorrüde. Eine Ärztin, ein Arzt und der Hund haben am 23.09.2006 den Abschluss zur Ausbildung als Therapiebegleithundeteam erworben.

Als weitere Rahmenbedingungen mussten die Einhaltung der Hygienebestimmungen in unserer Klinik, regelmäßige Tierarztbesuche, die Meldung beim Veterinäramt sowie eine bestehende Hundehaftpflichtversicherung und eine tiergerechte Haltung innerhalb der Klinik (siehe auch Große-Siestrup 2003) gegeben sein.

Der Therapiebegleithund wird auf allen drei Stationen (Vorschul-, Schul-, Jugendstation) in einer Tagesklinik für Kinder und Jugendpsychiatrie, Psychosomatik und Psychotherapie mit 36 Behandlungsplätzen eingesetzt.

Neben der täglichen praktischen Arbeit, wurden in den letzten Jahren hilfreiche Bücher zum Einsatz von Hunden in Pädagogik und Therapie verschiedenster Praxisfelder veröffentlicht (Koneczny 2006, Rütten 2007, Säger 2007, Vanek-Gullner 2007), welche gut in den Klinikalltag mit einbezogen werden können.

Der Arbeitsalltag unseres Therapiebegleithundes sieht wie folgt aus:

07.30-08.00 Uhr Beginn der ersten Therapieeinheit
08.00-09.30 Uhr Schlafen
09.30-10.15 Uhr Einsatz auf der Vorschulstation
10.15-12.00 Uhr Schlafen


12.00-12.45 Uhr Gemeinsame Mittagspause mit dem Team sowie Spaziergang mit angstbesetzten Kindern und Jugendlichen

12.45-13.30 Uhr Therapeutischer Einsatz auf der Schul- und Jugendstation

13.30-15.00 Uhr Ausruhen

15.00-15.20 Uhr Kuscheln, Lehren und Toben auf der Vorschulstation

16.00 Uhr Feierabend

Der Therapiehund Buster wird neben der Behandlung von Phobien, Depressionen und autistischen Störungen $u$. a. auch bei der Behandlung von Kindern mit einer Aufmerksamkeitsdefizit- und Hyperaktivitätsstörung sowie bei der hyperkinetischen Störung des Sozialverhaltens (ICD-10 F 90.o, F 90.1) eingesetzt.

Ein Programm für Tiergestützte Therapie bei aufmerksamkeitsgestörten Kindern setzten die Heilpädagoginnen Casaulta und Leung (2005) bei 5-7-jährigen Kindern ein. Sie fanden unter anderem ein verbessertes Aktivierungsniveau hinsichtlich der motorischen Unruhe sowie der Impulskontrollstörungen durch regelmäßige Berührungen des Hundes, und eine verbesserte Fremdund Selbstwahrnehmung, wenn der Hund z. B. müde war, die Kinder flüsterten. Außerdem förderte der Umgang mit dem Hund die taktil-kinästhetischen Erfahrungen der Kinder. Ebenso reflektierten Kinder erstmals etwas geschafft zu haben, wenn der Hund einer zuvor gestellten Aufgabe nachkommt.

Für den Einsatz der Tiergestützten Therapie fanden wir bei unseren ADHSPatienten unterstützend ebenfalls die Verbesserung der Wahrnehmung, der Impulsivität sowie der Motorik des jeweils gestörten Kindes oder Jugendlichen. Viele unserer Patienten erlebten erstmals eine wertfreie Annahme ihres Tuns, ohne gleich negative Erfahrungen zu sammeln.

Dabei ist der Selbsterfahrungsaspekt in der direkten Beziehung zwischen motorisch unruhigen Kindern und der Reaktion des Hundes besonders wichtig. Um so größer ist der Lerneffekt für unsere ADHS-Kinder, wenn sie sich motorisch ruhig verhalten, sich dem Hund nähern können, um ihn am Schluss streicheln zu können. Außerdem wird der Therapiehund nach der verhaltentherapeutischen Methode des operanten Konditionierens in unserer Klinik als positives Verstärkersystem mit einbezogen.

Im Einsatz auf unserer Vorschulstation wird der Hund in Kleinstgruppen bis max. 4 Kindern eingesetzt. Hierbei erfolgt ein strukturiertes Therapieprogramm, welches zuerst mit einem Stofftier geübt wird. Voraussetzung dafür ist eine anfängliche psychoedukative, an das Kleinkindalter angepasste Übung. Danach folgen Rollenspiele mit dem Stofftier und anschließend praktische Übungen mit dem Therapiebegleithund, welche dann abermals positiv verstärkt werden. Hilfreich können wir ergänzend das Buch „Ivanhoe im Kindergarten“ von Kathrin S. Becher (2005) empfehlen.

Auf der Schulstation, der Station, wo sich erfahrungsgemäß unsere meisten ADHS Patienten aufhalten, ist Buster ein fester Bestandteil des positiven 
Verstärkerplanes, welcher gut von den Kindern angenommen wird. Innerhalb des multimodalen Behandlungsplanes wird der Hund hier im Einzel- und Gruppensetting eingesetzt. Neben den zuvor genannten Erwartungen der Wahrnehmungs- und Positivitätsverbesserung und der besseren Steuerung der Motorik wird mit dem Hund die Leinenführung, die Kommandos, das Spielen, das gegenseitige Belohnen, aber auch die Tierpflege geübt. Das Tiere beruhigend auf motorisch unruhige Kinder im Gruppenkontext wirken, belegten auch die Studien von Naar et al. (2004) und Jalongo et al. (2004).

Die Therapieinhalte der Tiergestützten Therapie für unsere ADHS-Patienten sind in folgenden Schwerpunkten dargestellt:

- Gute Annahme des Therapiebegleithundes als positiver Verstärker

- Konzentrationstraining bei der Leinenführung im Straßenverkehr

- Körperliches Aktivitätsprogramm beim Toben mit dem Hund

- Selbstwertstärkung beim Ausüben von Kommandos

- Achtung und Rücksichtnahme in einer Gegenseitigkeit

- Vorurteilsfreie und positive Rückmeldung des Hundes

- Fein- und grobmotorische Beübung, Gleichgewichtsübungen, Koordinationsübungen

- Wahrnehmungstraining

- Spannungsabbau

- Wissensvermittlung

- Gegenseitige Rücksichtnahme gegenüber Mitpatienten innerhalb der Gruppentherapie mit dem Therapiebegleithund

- Teilen, Vermitteln, Angebote machen

Ebenso gelten die gleichen Schwerpunkte bei der Behandlung von Jugendlichen mit einem ADHS. Auch hier wird Buster als Verstärker hinsichtlich unseres multimodalen Therapiesystems eingesetzt. Weiterhin dient er hier zur Mobilisierung bei übergewichtigen Patienten und als Vermittler innerhalb der Psychotherapie bei bindungsgestörten sowie posttraumatisch belasteten Patienten.

Innerhalb des multimodalen Therapiekonzeptes in unserer Klinik ist die Tiergestützte Therapie ein fest integrierter Bestandteil geworden, der aufgrund der Größe von 36 Behandlungsplätzen weiter ausgebaut wird.

\section{Literatur}

Becher K.S. (2005). Ivanhoe im Kindergarten. Triga.

Casaulta T. und Leung E.Z. (2005). Erfahrungen mit einem Therapiehund. Schweizerische Zeitschrift für Heilpädagogik, 10 (2), 18-20.

Cassidy K., Webb L., Mc Keown D. und Stiles T. (1995). Evaluation of a companion animal program on a continuing care psychiatric unit. Perspectives 19, 15-20.

Frömming H. (2006). Die Mensch-Tier-Beziehung. Theorie und Praxis tiergestützter Pädagogik. VDM Verlag Dr. Müller.

Greifenhagen S., Buck 0. (2007). Tiere als Therapie. Kynos Verlag. 
Große-Siestrup C. (2003). Tierschutzgerechte Arbeit mit Tieren. In: Olbrich E., Otterstedt C. (Hrsg.) (2003). Menschen brauchen Tiere. Grundlagen und Praxis der Tiergestützten Pädagogik \& Therapie. Franckh-Kosmos Verlag.

Jalongo M.R., Astorino T. Und Bomboy N. (2004). Canine Visitors: The influence of therapie dogs on young children's learning and well-being in classrooms and hospitals. Early Childhood Education journal 32 (1), 9-16.

Koneczny M. (2006). Hunde im Kindergarten. Borgmann/Media.

Levinson P. (1962). „The dog as a „co-therapist“. Mental Hygiene 46, 59-65.

Naar L., Pilz E., Bräuer N. und Totrschal K. (2004). Immediate effects of exposure to a guinea pig on behaviour of pree-school children. In: People and animals: A timless Relationship, conference Handbook of the 10. International conference on Human-Animal Interactions.

Olbrich E., Otterstedt C. (Hrsg.) (2003). Menschen brauchen Tiere. Grundlagen und Praxis der Tiergestützten Pädagogik \& Therapie. Franckh-Kosmos Verlag.

Otterstedt C., Schwarzkopf A., Olbrich E., Rauschenfels C. (2004). Tiergestützte Aktivität, Förderung, Pädagogik \& Therapie, Definitionen und Qualitätsanforderungen. Unser Rassehund, Verband des Deutschen Hundewesens. 7, 16-17.

Prothmann A. (2007). Tiergestützte Kinderpsychotherapie. Peter Lang, Europäischer Verlag der Wissenschaften.

Röger-Lakenbrink I. (2006). Das Therapiehunde-Team. Kynos Verlag.

Rütten A. (2007). Tiergestützte Therapie. Für die Arbeit mit sprachentwicklungsgestörten Kindern. VDM Verlag Dr. Müller.

Säger I. (2007). Der Hund als pädagogischer Begleiter in der Frühförderung. VDM Verlag Dr. Müller.

Vanek-Gullner A. (2007). Lehrer auf vier Pfoten. Theorie und Praxis der hundegestützten Pädagogik. G und G Verlagsgesellschaft.

Vernooij M. A., Schneider S. (2007). Handbuch der tiergestützten Intervention. Quelle \& Meyer. 